\title{
UN COLEGIO NOCTURNO: UN TALLER SOBRE VIOLENCIA
}

\section{A NIGHT HIGH SCHOOL: A WORKSHOP ABOUT VIOLENCE}

\author{
Maurizia D' Antoni* \\ Mariángela Pluchino Arias**
}

RESUMEN

En la Universidad de Costa Rica, el estudiantado realiza 300 horas de Trabajo Comunal Universitario (TCU), con el propósito de devolver a la comunidad parte de lo que la universidad pública autónoma invierte en su formación. El tcu nro. 618 está orientado al rescate de modalidades educativas diferentes al colegio diurno "académico", buscando apoyar colegios nocturnos y técnicos profesionales que por un lado, tienen malas tasas de aprobación y por otro, muestran experiencias exitosas en el involucramiento escuela-comunidad. Se realizó un taller acerca de bullying en un liceo nocturno del casco metropolitano. El estudiantado involucrado expresa cierto conocimiento "ingenuo" de la vinculación entre violencia interinstitucional con la violencia estructural y expresa malestar ante la violencia verbal, la humillación y la burla, que se encuentra cargada de significados xenófobos, machistas y excluyentes.

PALABRAS CLAVE: ENSEÑANZA SECUNDARIA * EDUCACIÓN ALTERNATIVA * VIOLENCIA * EXCLUSIÓN * BULLYING

\section{ABSTRACT}

In the University of Costa Rica, the students perform 300 hours of community work (Tcu), in order to give back to the community part of what the public autonomous university invests in their formation. Tcu no. 618 is oriented to the rescue of educational methods different from 'academic' day school, seeking to provide support to nigh, professional or technical high schools that, on one hand, show poor approval rates and, on the other, show successful experiences in school-community involvement. A workshop on bullying took place in a night high school in the metropolitan area of San José. The students involved expressed a "naive" knowledge of the inter-linkages between structural violence with school violence and expressed rejection and unease at verbal abuse, humiliation and mockery, which is full of xenophobic, sexist and exclusive meanings.

\section{KEYWORDS: SECUNDARY EDUCATION * ALTERNATIVE EDUCATION * VIOLENCE * EXCLUSION *} BULLYING

\footnotetext{
* Escuela de Psicología y Departamento de Docencia Universitaria de la Universidad de Costa Rica (UCR). maurizia.dantoni@gmail.com
}

** Estudiante de Licenciatura en Psicología y Bachillerato en la enseñanza de la Psicología de la Universidad de Costa Rica (UCR).

mpluchino91@hotmail.com 


\section{INTRODUCCIÓN}

En la crisis educativa percibida en Costa Rica (Programa Estado de la Nación, 2013), el Ministerio de Educación Pública (мEP) declara querer promover la democratización de la educación pública. La lectura, a partir de la frecuentación de espacios educativos, es que las actividades realizadas hasta ahora no logran incidir significativamente en la estructura del sistema-escuela, así como se ha venido petrificando a lo largo del tiempo, en su funcionamiento y su lógica interna. Adicionalmente, el iv Estado de la Educación (2013) describe al Ministerio de Educación como un ámbito sumamente resistente a los cambios.

Uno de los aspectos sobre los cuales se centra la atención del iv Estado de la Educación es la "gran diversidad" atribuida a los planes de estudio en la secundaria y su consiguiente falta de evaluación (2013: 168). El espacio que el documento le otorga al desempeño de la educación general básica y al ciclo diversificado abarca, entre otros, todo el tercer capítulo y un apartado se dedica específicamente, a los planes de estudio en la secundaria y a su evaluación (2013). El informe discute datos sobre la educación técnica y se enlaza la discusión al Instituto Nacional de Aprendizaje (INA) y a la necesidad de ampliar la cobertura de la educación parauniversitaria, para llegar luego al apartado sobre educación indígena, sumida en un "rezago histórico" (Programa Estado de la Nación, 2011 y 2013: 174).

Una de las modalidades educativas que más "sufren" en el ámbito institucional en la actualidad es el colegio nocturno: modalidad tristemente conocida por los escasos índices de promoción de sus estudiantes ${ }^{1}$, es presentado como un tipo de colegio sobre el que se necesita discutir y que se necesita evaluar (Programa Estado de la Nación, 2013). Sobre la necesidad de evaluación, se señala que la discrepancia con esos documentos oficialistas reside en la centralidad que sería necesario atribuir al juicio de

$1 \quad$ La aprobación en los colegios nocturnos es de un $41,4 \%$, promedio inferior a la de los colegios diurnos que se definen como "académicos" (72,8\%) (Programa Estado de la Nación, 2013: 176) actores y actrices de la actividad formativa $y$ de la comunidad, relacionados con los beneficios que la modalidad educativa aporta, privilegiando esa opinión sobre el enfoque centrado en los datos estadísticos.

Por todo lo anterior, se establece la necesidad de abrir un debate para el rescate de modalidades educativas surgidas o implantadas por diferentes administraciones, fruto de diferentes momentos políticos. En efecto, algunas de esas modalidades se han convertido en proyectos exitosos, interesantes o necesarios en el ámbito de la educación secundaria o de la educación ofrecida a la juventud, a la par de la educación secundaria diurna regular (D’Antoni y Soto, 2010).

Se toma como una indicación, por ejemplo, el enorme crecimiento de la matrícula de los Institutos Profesionales en Educación Comunitaria (IPEC) $)^{2} y$ de los Centros Integrales de Educación para Adultos (CINDEA) que investiga el iv Estado de la Educación (2013).

Se tomó en consideración un taller realizado en un colegio nocturno del área metropolitana del que se reserva el nombre, en el marco de las acciones planeadas por un Trabajo Comunal Universitario (тс)), donde se había solicitado un taller sobre "bullying", lo cual permitió abarcar un tema más amplio sobre violencia y violencia estructural.

\section{APORTES A LA ACCIÓN SOCIAL}

A través de las actividades del тси-618 de la Vicerrectoría de Acción Social, en la Universidad de Costa Rica, se intenta aportar acciones $y$ reflexiones que apoyen una visión diferente de modalidades educativas que no sean el colegio diurno tradicional.

$2 \quad$ El IPEC es una modalidad ofrecida por el Ministerio de Educación Pública costarricense. La oferta educativa del IPEC se da en educación secundaria, donde las lecciones se reciben en forma modular, por bloques de materias, se aprueban una a una hasta completar los diferentes niveles del bachillerato, como ventaja adicional junto con el bachillerato obtienen una formación técnica. En el CINDEA se puede optar por conseguir bachillerato de secundaria o para brindar formación técnica de forma libre (Leiva, 2009). 
Este тcu denominado "Promoción de inclusión y participación democrática en la institución escolar costarricense. Fortalecimiento de modalidades institucionales alternativas", se crea con la intención de aumentar la visibilidad y apoyar el fortalecimiento de experiencias educativas en la secundaria que beneficien a la comunidad, las cuales surgieron como expresión de necesidades específicas o como una propuesta institucional.

La visión oficialista sobre la oferta en la educación superior, no coincide con el enfoque que se da en la educación general básica, debido a que la oferta formativa para adolescentes es confusa $y$ ofrece un número exagerado de modalidades:

El análisis realizado puso de manifiesto una serie de problemas. En cuanto a la viabilidad de los planes de estudio, determinó que si bien algunos buscan dar respuesta a necesidades particulares de la población y de la sociedad, no se implementan con los recursos y el apoyo requeridos, lo que impide que se logren los objetivos y las expectativas de la comunidad. Sobre la vigencia de los programas, no se encontró evidencia de que estos se sometan a estudios periódicos, científicos y sistemáticos para su actualización (Programa Estado de la Nación, 2013).

El caso del ipec-cindea de Cartago ha sido analizado con la intención de rescatar su historia como experiencia exitosa en el panorama institucional actual (D'Antoni y Soto, 2010), con lo cual se destaca la existencia de ámbitos donde se puede trabajar eficientemente $y$ de manera diversa con el estudiantado, atendiendo sus necesidades $y$ aspiraciones en el área formativa.

Es importante destacar que los espacios más excluidos de la institución escolar son los que reciben menos dinero, con las aulas más deterioradas y en los lugares más distantes. En este sentido, se hace referencia a los IPEC-CINDEA $y$ a espacios como las Aulas Abiertas, Aula Recurso, Aula Integrada, Aula de Trastornos Emocionales y Pre vocacionales.
Por esta razón, este tcu se ha dedicado al apoyo de experiencias valiosas en opciones educativas no estándar, visibilizar sus alcances y proyectos, empoderar a las personas que estudian en estos espacios, impulsando su participación democrática y la inclusión escolar. Asimismo, se busca impulsar a que esas personas "salgan" de la institución y de las aulas para darse a conocer $y$ participar de la vida comunitaria y productiva con las herramientas obtenidas por este medio.

En la espera de la gran reforma de la escuela de la que se habla en toda América Latina, se optó por empezar a destacar lo valioso en lo existente. Se han realizado acciones educativas que pueden dar resultados, si se deja de pensar en la opción educativa estandarizada como la única válida, mientras que la oferta de modalidades diferentes se convierte en vehículo de mayor exclusión. Por ejemplo, se pretende destacar la incongruencia de que un colegio nocturno - creado para permitir el acceso a la educación a las personas estudiantes que trabajan o cuidan a niños(as) - sea visto como una de las modalidades con el índice de promoción más bajo (Programa Estado de la Nación, 2013).

En el aula, la intención es concertar, desarrollar y promover estrategias psicoeducativas para la inclusión y la participación democrática en modalidades educativas alternativas de las aulas públicas costarricenses, con la finalidad de lograr el empoderamiento de sus actores.

A partir de esto, se trabaja con los actores de la educación, construyendo actividades educativas definidas para la promoción de la cultura política $y$ de la participación democrática en la institución escolar. A la vez, se pretende cooperar con la institución para la vinculación de niños(as), adolescentes $y$ docentes de la modalidad educativa alternativa.

Con el personal docente de la institución, así como, directores(as) y supervisores(as), el objetivo es motivar el fortalecimiento de la oferta de modalidades diversas en la escuela y colegio costarricense, mediante la capacitación de maestras(os) y profesoras(es).

Finalmente, la intención hacia la comunidad, incluye divulgar y promover bases teóricas, 
historia y acciones realizadas por las modalidades educativas alternativas, a través de la participación en ferias vocacionales, programas de radio, programas municipales, lugares en red o la ubicación en otros espacios comunitarios viables.

Asimismo, se pretende un espacio, en el cual se realizarán acciones dirigidas a la concertación con autoridades locales o nacionales de un concurso, un premio o un reconocimiento con el fin de empoderar a las personas $y$ visibilizar el trabajo realizado en un espacio educativo alternativo.

\section{ALTERIDADES Y VIOLENCIA}

La reflexión que se ha realizado acerca de los espacios educativos "alternativos" a esos colegios diurnos que el Ministerio de Educación Pública de Costa Rica llama "académicos", permite colocar esa educación de adolescentes $y$ adultos en un espacio de alteridad, ante la homogenización que el sistema educativo parece propiciar.

Según Baquero (2001) existiría una versión débil y una fuerte sobre el pensar la alteridad, que presentan una distinción con respecto a la sensibilidad hacia el tema de la diversidad. La débil se limitaría a presentar al diverso como una alteridad que emerge sobre la homogeneidad; por ejemplo, al coeficiente intelectual o a la lengua materna de la persona escolarizada. Además:

... la homogeneidad puede pensarse también con respecto a las metas: un mismo logro relativamente homogéneo sitúa a los aprendices en un punto de diversidad. En verdad, parece plantearse la existencia de un conjunto o tipo relativamente homogéneo de sujetos en tanto su punto de partida o características son relativamente comunes $y$, por tanto, su distancia a los logros buscados o los medios a través de los que estos piensan conseguirse resultan también relativamente homogéneos. Es probable que la lógica de la escolaridad "común" este fuertemente impregnada de esta concepción de lo diverso (Baquero, 2001:43).
Por lo consiguiente, emergería una visión difusa de la diversidad, entendida como distancia "especial" de puntos de partida y de niveles de logro relativamente comunes.

En cambio, una versión más "radical" sobre diversidad entendería que todo enunciado de homogeneidad relativa se involucra con la problemática inclusión/exclusión y a través de esta vinculación, tiene alcances políticos. Se llega a pensar que el concepto de diversidad implica a toda la población escolar, más allá de la construcción de una ilusión de homogeneidad: todos y todas estamos en la "diversidad".

De esta manera, la diferencia viene a significar: "... aquellos elementos singulares - o propios de una identidad cultural — de la subjetividad bajo la perspectiva de atender a su propia identidad más allá de las intenciones homogeneizadoras de las prácticas de enseñanza" (Baquero, 2001: 43).

Para Salinas (2002), se han aplicado políticas de homogeneización y asimilación cultural en las escuelas de todos los países latinoamericanos. El resultado ha sido una trayectoria escolar caracterizada por el fracaso escolar, con particular énfasis en las áreas indígenas de América: de allí que se podría asumir la temática de la homogeneización en lo educativo como una de las formas de violencia estructural que llevan a la expulsión.

La construcción de una comunicación educativa más simétrica y adecuada a una realidad intensamente diversa $y$ plagada de desigualdad de nuestro continente se puede conseguir a través de un proyecto que tome en cuenta la interculturalidad. La propuesta para responder a la homogeneización en la educación pública es una educación que tenga profundo arraigo en la cultura propia $y$ a la vez, que entable un diálogo crítico entre tradiciones culturales diferentes (Salinas, 2002).

Si la homogeneidad fuera un proyecto defendido en la institucionalidad educativa, también se convertiría en criterio de exclusión y como tal, direccionaría a la violencia institucional, aquella que discrimina y expulsa.

Gómez, Leigh y Nova (2012) vinculan la desigualdad en el sistema educativo con la violencia, estudiando como el currículo promueve la desigualdad y gestiona la violencia en 
la escuela. Según las autoras, que se refieren a la experiencia de Chile, el contexto en el que se desarrolla el actual debate sobre la violencia en las escuelas es un contexto de creciente fragmentación, con la violencia social como una de sus principales expresiones.

Citando la capacidad ideológica que tiene el currículo oculto ${ }^{3}$ e influenciar a las personas, las autoras rastrean los ámbitos que se utilizan para ese propósito:

Las prácticas, los procedimientos, las reglas, las relaciones, las estructuras, el discurso, las actitudes de los profesores, el lenguaje, estructura social de la clase, el ejercicio de la autoridad por parte del profesor, las medidas disciplinarias, los sistemas de evaluación y las prioridades curriculares (Gómez, Leigh y Nova, 2012: 179).

De esta manera, en oposición a la manipulación y a la homogeneización, las autoras plantean que las tareas educativas deben tener como objetivo el de lograr la identificación del sujeto con las identidades sociales válidas y legítimas, dentro del orden simbólico. En ese sentido, la enseñanza debe adquirir un carácter de permeabilidad a los contenidos personales y dejar caminos abiertos para la expresión de significantes personales. De no ser así, existe el riesgo de perder los planos únicos e irrepetibles de la experiencia personal (Gómez, Leigh y Nova, 2012). La violencia concreta y la violencia simbólica permean de esta forma espacios escolares homogeneizados, indiferentes a la diversidad y a los derechos de las personas.

Es preocupante el mensaje de desconocimiento hacia "el otro" como semejante a través de la violencia cotidiana presente en la institución escolar (Gómez, Leigh y Nova, 2012), mientras que la propuesta de las autoras es un currículo como construcción socio cultural, la

3 El currículo oculto es aquel conjunto de normas y valores que son implícitos, se enseñan eficazmente en la escuela pero no se suele hablar en las declaraciones de fines u objetivos de los profesores $y$ profesoras (Apple, 2008). atención a la interculturalidad y la diversificación curricular.

\section{UN TALLER SOBRE VIOLENCIA}

Durante el тси-618, se realizaron talleres sobre temáticas variadas en un colegio nocturno en la localidad de San Pedro. Se desarrollaron talleres de capacitación para docentes, con el objetivo de conocer $y$ discutir las políticas que establecieron esa modalidad no tradicional y sus contenidos. Entre las herramientas de trabajo utilizadas, se contó con plataformas electrónicas o Facebook, entre otras.

Con el objetivo de planear y preparar los talleres, los y las estudiantes del тcu establecieron un encuentro en el Colegio al inicio del año lectivo, con el fin de investigar acerca de los intereses y necesidades manifestadas por la población estudiantil.

Entre las temáticas sugeridas para ser abordadas se destacó la de violencia y de manera específica, el bullying (en inglés).

Al inicio del taller se explicó acerca del consentimiento informado, se leyó el contrato y se pasaron dos copias impresas del mismo entre los y las participantes, quienes dieron su aprobación oral y escrita al uso de la información recolectada como parte de un proyecto de investigación.

Al igual que en otras experiencias de investigación (D’Antoni y Gómez, 2013), se observó en ese momento que los y las estudiantes habían sido expuestas a explicaciones y a trabajo formativo que introducía el concepto de bullying. Además, identifican la temática como una de las problemáticas más "graves" en el sistema educativo nacional; sin embargo, no realizan reflexivamente la vinculación del tema de la violencia en la institución con la violencia en general o con la violencia estructural.

Tampoco se discute la pertinencia del uso del concepto de bullying en inglés, cuando existen términos en español que describen este fenómeno como por ejemplo, la palabra matonismo, lo cual se explicará posteriormente.

Entre otros talleres que se realizaron en el colegio nocturno por parte de los y las estudiantes del тcu, se trabajó orientación vocacional dividido en tres sesiones, en las cuales 
se observó acciones relacionadas con el tema de la violencia: que la vinculación de los y las estudiantes en el aula se caracterizaba por una serie de marcadas bromas, risas, chistes, manifestaciones en voz alta de aprobación o desaprobación de las opiniones de los pares y un elemento frecuentemente identificado por los $y$ las costarricenses en su manera habitual de vincularse: el choteo ${ }^{4}$.

Una de las manifestaciones de esto que se podría considerar un acto de discriminación, enmascarado con el humor, emergió en una parte del taller donde una de las coordinadoras de la actividad explica los procedimientos que las personas extranjeras deben llevar a cabo para matricular en la ucr. Ante esta explicación, uno de los participantes comenta riendo que su compañero de al lado es inmigrante y que debe estar interesado en esta información. La facilitadora detecta este comentario como una burla, ante lo cual se muestra seria y continúa explicándoles en qué consiste el procedimiento. El participante aludido por el comentario insiste en decir, igualmente en tono de burla, que él es extranjero, francés hijo de italianos, lo cual no es cierto.

Se considera esto como un acto interesante porque, si se somete a un análisis crítico de contenido de la broma, se podría dejar entrever que hay una especie de jerarquía en función a la nacionalidad: es decir, el chico que hizo la primera intervención quería bromear respecto a la condición extranjera de la persona, "achacándole" al otro una nacionalidad probablemente nicaragüense, cuando esto no era cierto. A su vez, el chico aludido no solo evade la broma con humor, sino que se identifica con dos nacionalidades que dentro del imaginario podrían ser asociadas con

$4 \quad$ El choteo es entendido como una de las formas críticas que utiliza la sociedad costarricense (Gutiérrez, 2013). La función es la de mofa o de burla en clave sarcástica, no solo el otro como sujeto cultural sino hacia asuntos como la política, la religión y la economía. Gutiérrez (2013) establece que el choteo costarricense es una temática que merece ser estudiada como característica sociocultural del ser costarricense. La temática se encuentra; sin embargo, no solamente en Costa Rica, sino que se estudia en otros países de América latina. mayor "distinción". Se señala el valor de esta reflexión, al ser este ámbito investigativo una vinculación mayormente auto etnográfica, más que a un pretendido "cientificismo".

Por esta razón, se considera importante señalar que la violencia, en este caso disfrazada de burla, está presente en muchas de las acciones que se dan en el marco de la actividad formativa; toda la situación indicada, mediada por una serie de códigos intrínsecos y compartidos entre los participantes y espectadoras de la broma, muestra una naturalización de los argumentos y discursos de la exclusión (Calvo y Susinos, 2005-2006), una colectivización de la burla, los marcos de estigmatización compartidos y asumidos silenciosamente.

\section{ENTREVISTA CON UN GRUPO DE ESTUDIANTES DE UN LICEO NOCTURNO}

Al inicio del año lectivo de las instituciones públicas, el тсu organizó una actividad de discusión con un grupo de estudiantes del colegio nocturno, con la finalidad de expresar las temáticas de interés a trabajarse en los talleres.

La institución educativa, a través de dos profesores y del director, solicitó abarcar directamente el concepto de bullying, debido a que el Ministerio de Educación Pública le solicitaba a todas las instituciones de enseñanza una intervención específica sobre esa temática.

Uno de los talleres sobre violencia, se realizó en una fecha previa a las vacaciones de medio año, para poder estratégicamente mencionar el tema del abandono escolar, que repercute en escuelas y colegios después de este periodo (Programa Estado de la Nación, 2013).

A continuación, se decidió presentar el taller (originariamente preparado como grupo focal) de una manera particular, evidenciando la vivencia de cada una de las dos investigadoras: las dos señalaron en efecto intenciones $y$ momentos emergentes distintos.

Se presenta como un momento de exploración en clave autoetnográfica que va más allá del análisis crítico del discurso. García (2012) rescata el método autoetnográfico en el ámbito de la investigación educativa, para aportar al estudio de las didácticas específicas de las artes. Se trata de una metodología donde 
la persona investigadora se incluye en el cuadro total $y$ lo hace con plena conciencia de que sus vivencias, representaciones $y$ opiniones afectan $y$ son afectadas por el proceso investigativo.

El grupo participante en el taller se conformó por 32 estudiantes de un liceo nocturno en San Pedro de Montes de Oca, el día 20 de junio de 2013, a las 18h 30.

Seguidamente, se encuentran las bitácoras del taller, como material importante para el análisis que toma en cuenta la metodología autoetnográfica mencionada anteriormente.

\section{BITÁCORA UNO}

La mayoría de las personas presentes comentaron haber iniciado sus estudios en un colegio diurno y que posteriormente, fueron trasladados por diversas razones a la modalidad nocturna, solamente cuatro, como excepción, comentaron haber iniciado sus estudios en un colegio nocturno.

Algunas de las razones que los llevaron a cambiar la modalidad diurna por la nocturna fueron:

$\diamond \quad$ Razones laborales: comenzaron a trabajar en un horario diurno $y$ los horarios con la institución educativa y el centro laboral no coincidían.

$\diamond \quad$ Edad: los $y$ las estudiantes sobrepasaban la edad máxima requerida para permanecer en la modalidad académica diurna de la institución educativa.

$\diamond \quad$ Conflictos personales: conflicto por el cuido de algún familiar enfermo y maternidad en el caso de las mujeres.

$\diamond \quad$ Conflictos en el colegio diurno: problemas de narcotráfico, relaciones interpersonales entre compañeros o relaciones con los y las docentes.

La elección de matricularse en un liceo nocturno no se muestra como primera opción ni corresponde a intenciones concretas que indiquen un interés particular por esta modalidad en el grueso de su población usuaria.

Ante la pregunta respecto a la definición de bullying, se mencionaron términos como agresión física, verbal o de índole psicológica.
Se mencionó el colegio como principal espacio donde se da esta problemática, pero también una intervención se refirió a otros contextos donde se da, más allá del espacio de la institución educativa.

Se comentó haber recibido esta información de las noticias y del Internet, incluso un estudiante mencionó que el bullying venía de Estados Unidos, ante lo cual otro respondió que aquí también existía ese fenómeno. Por lo tanto, es interesante ver como se identifica el concepto bullying como una imitación cultural de una producción extranjera.

$\mathrm{Al}$ indagar sobre las formas de bullying presentes en esta institución, si bien admitieron que era un fenómeno presente, comentaron que en la población adulta, las situaciones de burla entre compañeros eran menos ofensivas, puesto que ya las personas tenían una personalidad consolidada que disminuía el daño percibido ante las mismas. Explicaron que desde su punto de vista, el bullying era un fenómeno que afectaba principalmente a escolares adolescentes $y$ niños(as) por la vulnerabilidad que estas etapas suponen en su formación. Asimismo, el grupo de estudiantes admitieron haber sido testigos y protagonistas de situaciones de bullying en otras instituciones educativas.

Los y las participantes se interesaron por lo que identificaron como bullying del personal docente hacia el estudiantado, para denunciar una serie de situaciones en esa $y$ otras instituciones educativas que involucraban maltratos en esta dirección. Sin embargo, uno de los estudiantes comentó que la mejor alternativa ante una situación de este tipo era no darle importancia $y$ no denunciarla.

Se habló de que por una disputa de celos, un estudiante le apuntó con un revólver a otro estudiante, el cual no volvió a la institución. Este acontecimiento abre a la discusión del uso de armas dentro de la institución, mientras que los y las participantes bromearon respecto a la facilidad de introducir armas al liceo.

En este punto de la actividad se presenta el director de la institución acompañado de una funcionaria supervisora del MEP, la cual interrumpe la actividad para hacer un saludo, entra $y$ hace una breve consigna motivacional, 
menciona su experiencia personal, la de su hija, ambas estudiantes de colegios nocturnos, finaliza su intervención, no sin antes mencionar a Dios $y$ a la familia.

Al retomar la entrevista y ante la temática de violencia como un concepto más general que se puede trasladar a la vida cotidiana, se preguntó si ellos y ellas se consideraban expuestos ante situaciones de violencia cotidiana, ante lo cual la mayoría respondió que sí, identificando esos escenarios como el servicio al cliente de las instituciones públicas. Un estudiante que a su vez es funcionario de la fuerza pública comentó la alarmante cantidad de denuncias de violencia doméstica por parte de mujeres, asimismo, una chica comentó experimentar situaciones de agresión en su hogar por parte de su padre y de su tío.

Es importante destacar que anterior a la intervención de esta chica, la participación por parte de las mujeres en la entrevista fue muy escasa, sobretodo en la temática de violencia, que a su vez es una temática que afecta en gran medida a la población femenina, posicionándose como una de las más afectadas en esta disposición patriarcal de la organización social (Guedes, García-Moreno y Bott, 2014).

Las principales razones desencadenantes de la violencia que los y las participantes identificaron fueron la falta de recursos económicos, el consumo abusivo de drogas y alcohol. Una chica concibió la violencia como un ciclo intergeneracional, en el cual familias de abuelos y padres agresores, serán propensas a criar chicos $y$ chicas agresores(as).

El tema religioso suscitó no pocas reacciones, varias de las personas participantes estuvieron de acuerdo con que la religión suponía un buen amortiguador para disminuir la violencia; sin embargo, algunos dieron excepciones puntuales como: "- -en mi casa éramos siete e íbamos todos los domingos a misa y ustedes no saben todos los pleitos que teníamos en casa", refiriéndose a que la religiosidad no era suficiente para evitar la violencia.

Uno de los participantes se refirió a la importancia de educar con un estilo autoritario para instalar esquemas de vinculación basados en el respeto y la obediencia: "-mi abuela decía: en una mano amor y en la otra rigor"; no obstante, el mismo participante denunció también una cierta disconformidad con el modelo de masculinidad que se maneja en la sociedad costarricense, pues dice que desde pequeños, a los chicos varones, no se les premia por demostrar sus sentimientos, sino que se les ridiculiza, asociando la muestra de sentimientos con una sexualidad homoafectiva, lo cual también se identifica como socialmente inaceptable.

\section{BITÁCORA DOS}

Las investigadoras son presentadas por el profesor de Matemáticas, quien ya había anunciado su llegada. Ese día se canceló un examen de Física, por lo tanto, el profesor de Español permitió que el grupo de undécimo participara en el taller. Se concentran de esta manera dos grupos del liceo nocturno: décimo y undécimo. En total participaron 32 personas. Es un grupo muy grande para un grupo focal; no obstante, se debía seguir con la actividad y convertirla en un taller.

El profesor realiza la presentación, para proseguir con el consentimiento informado, $y$ pasar las hojas con el "contrato". Las personas las van leyendo y las firman mientras se continúa con la actividad.

Se realiza una explicación sobre el objetivo del тси y de las actividades que están haciendo los y las jóvenes universitarios(as) matriculados en el proyecto (la organización de la biblioteca, las clases de apoyo, entre otras) y se propone la realización de un partido de fútbol.

De las "cápsulas informativas" que se había planeado llevar sobre las temáticas de violencia estructural y alfabetización crítica, se logró un espacio para varias. Por ejemplo, sobre alfabetización crítica y hablando de un tema de actualidad en los periódicos, se comenta sobre la carretera a San Ramón y el intento de corrupción con el presupuesto para su realización, ligado con una empresa constructora extranjera ${ }^{5}$. La investigadora argumenta que a partir de un interés común y una unión de grupo, la gente de San Ramón logró detener

$5 \quad$ Referencia sobre el tema se puede encontrar en el artículo de Rivera (2013). 
el proyecto; se continúa manifestando que de igual manera, los y las estudiantes presentes tendrían el interés común de mantener abierto el colegio, amenazado de cierre.

Por otro lado, se comenta sobre el tema de individualismo y refutan la comparación: reivindican que en su caso no tienen un interés común o no están dispuestos y dispuestas a unirse y compartir. Según el criterio de las investigadoras, no se comparte esta posición con la cual se abre la actividad, pero las personas que la expresan están decididas y el grupo en general lo aprueban.

Otro aporte de los participantes fue sobre las razones de retirarse de un colegio diurno normal. Algunos(as) dicen que se retiraron por maternidad o por otras circunstancias. Por otro lado, el tema de la vinculación de grupo se retoma a partir de la reacción y preguntas de una de las investigadoras sobre un joven estudiante que se retiró por la necesidad de trabajar. A partir de esta situación, surge la interrogante: ¿será esta una necesidad compartida por estudiantes del liceo nocturno?

Se interroga acerca de las razones que han influido a matricularse en un liceo nocturno, por lo cual uno de los estudiantes relata que en su anterior colegio vendían drogas y algunos de los profesores estaban confabulados, él denunció la venta ilegal en el colegio con un profesor que lamentablemente estaba de acuerdo con el proveedor de drogas, por esta razón, el estudiante decidió irse del colegio diurno.

En este momento, se inserta otra "cápsula informativa" sobre el narcotráfico, debido a que la violencia que se vive en las comunidades y los barrios - vinculada por la prensa con el trasiego de drogas - es una realidad conocida, pero tiene también una articulación con un marco más amplio de sus manifestaciones específicas que se presencian.

De la misma manera, el tema de la violencia en general se vive en el plano familiar $y$ local, a la vez se enlaza con situaciones institucionales, políticas y sociales. En el taller, el tema de la penetración del narcotráfico a Costa Rica se introduce, con el ejemplo de la tasa cambiaria del dólar con respecto al colón $y$ la posibilidad de que haya una relación entre la liquidez que el narcotráfico dispone y la tasa cambiaria del dólar, la cual se ha mantenido desde hace uno cinco años, alrededor de los 500 colones por dólar, a pesar de las maniobras del Banco Central.

El estudiantado manifestó conocer a las personas que venden drogas y personas que consumen; no obstante, esta problemática podría ser vista desde otra óptica: no solamente la persona que consume y que yo conozco (o tal vez yo mismo o misma esté haciendo uso de sustancias), sino la idea es empezar a discutir sobre la incidencia que tiene el narcotráfico en una dimensión colectiva, el impacto sobre la sociedad, la cultura y la economía.

En ese momento de la actividad, se obtienen comentarios breves; en general, se tiene una audiencia interesada, respetuosa y participativa.

Anteriormente, al entrar a la institución, se observó siete $\mathrm{u}$ ocho asesores $y$ asesoras del Ministerio de Educación Pública, sentados(as) en fila fuera de la secretaría. En este momento de la discusión, entra una asesora acompañada por un profesor y el director del colegio. El grupo de estudiantes la saludan con un "buenas" fuerte e irónico, para evidenciar que ella no había saludado, por lo cual ella responde que al menos le den tiempo de entrar para saludar. La asesora empieza con una pequeña charla motivacional diciendo que ella misma se graduó de un colegio nocturno, así como sus hijas, una de las cuales está ahora en la universidad. Asimismo, realiza menciones a Dios y la familia como el eje central de la sociedad, posteriormente se retira.

Se resalta que quienes participan y reaccionan más en el taller son en general los hombres, de los cuales, cuatro responden las preguntas con mayor frecuencia. De las mujeres, cinco de todo el grupo toman la palabra; un par de ellas solamente realizan un comentario y una que llegó tarde se sienta en primera fila para trabajar un examen de inglés con un compañero.

Cuando se trabaja el tema de la violencia y la violencia estructural, llama la atención el relato de una estudiante, quien comenta sobre la violencia doméstica que se vive en su casa, 
con un padre alcohólico que le pega a la madre; asimismo, un tío que patea la puerta y los amenaza cuando está borracho. Es angustiante no poder dar otra respuesta que agradecer la confianza que tuvo al contar su historia.

Otro tema que surge es sobre la religión, a partir de un comentario de un estudiante que afirma que la causa de la violencia es que las familias no tienen a Dios y que en su caso, su familia "está con Dios", por lo cual no hay violencia doméstica, opuesto a lo que sucede con los ateos. Otro estudiante le responde señalando que su familia va a la iglesia todos los domingos $y$ a pesar de esto, se pegan y pelean fuerte $y$ constantemente. Posteriormente, interviene una investigadora quien recalca que es atea, no obstante, lo importante es que las personas compartan una espiritualidad.

En la actividad se apoya en cierta manera la opinión del primer estudiante al decir que si falta espiritualidad es más probable la violencia, sin embargo, la espiritualidad puede tener diversas "direcciones". No obstante, no se apoyó el comentario de "acusar" a las personas de ser violentas por no tener a Dios.

Fue una situación tensa luego de la historia de la muchacha víctima de violencia doméstica; sin embargo, surgió una repuesta inmediata que manifestó como no necesariamente las prácticas religiosas evitan la agresión.

Es evidente que los ambientes familiares y comunitarios están expuestos a situaciones de violencia, lo cual es parte de la vida cotidiana de casi todas las personas de este grupo de investigación. El estudiantado muestra que tiene sus propias reflexiones y respuestas sobre las causas que la provocan: el machismo, el alcohol y la falta de apego a la religión. Por otra parte, las razones más estructurales como la exclusión educativa, el retraimiento de los servicios del Estado y la desigualdad, son problemáticas que manifiesta conocer, pero que ven mucho más desdibujadas como posibles causas de la violencia. Por esta razón, las investigadoras intentan vincular estas problemáticas en la discusión y evidenciar el papel que desempeñan en la temática de la violencia.
Con respecto al ambiente institucional que se presenta en este colegio nocturno, existen diversas historias sobre violencia, las que se definen en el iv Estado de la Educación (2013), como "violencia básica". En la entrevista grupal, se comenta persistentemente sobre la presencia de crueldad verbal con la finalidad de herir $y$ de humillar continuamente. De esta manera, se exponen comentarios acerca de lo doloroso que es y como en algunos casos, puede ser motivo de alejamiento del estudio. Las conclusiones de quienes facilitan la actividad es que hay una carga importante de dolor, asociada a la denuncia de estos actos por parte de los $y$ las estudiantes.

De esta manera, al abordar el tema de abandono escolar, se les solicita que observen si algún(a) compañero(a) no vuelve a clases después de las vacaciones de medio año.

Una de las participantes concluye comentando que ella se encuentra desde hace cuatro años en el colegio, ya que debió trasladarse de otra institución por diversos problemas. En esta institución nadie la ha molestado $y$ que se sorprende al escuchar los comentarios de los demás sobre la violencia en el colegio.

Se finaliza retomando el tema del partido de fútbol y programando actividades para después de vacaciones.

\section{VIOLENCIA EN LOS COLEGIOS: "NUESTRO" LICEO Y EL ESTADO DE LA EDUCACIÓN}

En el iv Estado de la Educación en el título del apartado dedicado a violencia en los colegios, se afirma que es un problema persistente pero no generalizado - asumiendo una posición similar a la del grupo de estudiantes del colegio nocturno y al contrario de estas autoras-, el cual separa las problemáticas de la pobreza, la desigualdad y la exclusión social, del fenómeno de violencia en las instituciones escolares. A la vez, el título citado "minimiza" el problema recalcando que no es "generalizado".

En efecto, en el texto se dice que un 25\% de los y las estudiantes de los centros educativos reportan haber sido víctimas de la violencia más común, que consiste en "burlas y humillaciones constantes"; un 16\% afirman haber sufrido daños a la propiedad, hurtos o robos; 
finalmente, un $10 \%$ señala haber sufrido amenazas constantes, hostigamiento, discriminación o rechazo, mientras que "solamente" entre el $3 \%$ y el $7 \%$ de la población estudiantil ha sido víctima de “... los actos más violentos que incluyen el uso de cuchillos o armas de fuego $y$ hostigamiento o agresión sexual" (Programa Estado de la Nación, 2013: 180).

En este documento, se describe una escalada de violencia que permite reflexionar sobre una cultura de violencia o en un problema estructural. Aunado a esto, se hace referencia a la violencia ejercida entre estudiantes $y$ entre docentes-estudiantes. El mayor número de situaciones violentas en las instituciones escolares se sitúa en el aula.

Por las razones antes expuestas, este taller refleja esta visión, con la diferencia de que la violencia verbal, el choteo, las humillaciones $y$ las bromas con doble sentido, hieren y pueden causar abandono escolar, principalmente en las personas jóvenes y menos estructuradas. Existe una perspectiva protectora del colegio nocturno como lugar al cual se puede acudir cuando otras modalidades no han funcionado.

En el estudiantado existe una incipiente conciencia de las causas que provocan la violencia: la drogadicción, el machismo y el alcoholismo; no obstante, existe una escasa capacidad de mapearlas $y$ de crear cadenas causales o modelos explicativos más complejos para estos fenómenos.

Los y las participantes ubican la violencia en su propia historia de exclusión educativa, que llevó al "refugio" del colegio nocturno; ven a este último como un lugar al que llegan con menores ingenuidades $y$ mayor capacidad de defenderse, una institución menos violenta; no obstante, se dan situaciones violentas y amenazas con arma de fuego.

La violencia verbal, la humillación y la burla llegaban a una respuesta individualista, que a su vez los y las deja más débiles frente a una institución expulsora e injusta.

Es el individualismo, exhibido como inevitable consecuencia de la agresión de los demás, el rasgo que se acoge con mayor pesimismo y que emerge de una trama institucional de violencia y exclusión social para manipular las posibilidades de persecución de objetivos comunes.

\section{HACIA UNA VISIÓN CRÍTICA DEL CONCEPTO DE BULLYING}

La discusión y problematización sobre el fenómeno de la violencia en las escuelas, en su versión anglicismo crudo y popular, bullying, nos interpela como investigadoras de la temática.

¿Es acaso este préstamo de la lengua de nuestros homólogos del Norte una casualidad lingüística? ¿O hay detrás de esto una intencionalidad paradigmática?

Se refiere al bullying 6 para designar cualquier conducta de agresión física, verbal o psicológica ejercida por un o una estudiante hacia algún o algunos miembros del grupo de pares y que tenga consecuencias negativas sobre la salud o el bienestar integral, en este caso, de las personas estudiantes (Paredes, Álvarez, Lega y Vernon, 2008).

Si bien, en este ensayo se pretende delimitar a un contexto escolar específico, se considera que para llegar a una verdadera comprensión de este fenómeno, es necesario hacer una aproximación más panorámica y tomar en cuenta factores más macro que podrían estar incidiendo directa o indirectamente en los índices de bullying y sus distintas manifestaciones.

Para hacer referencia a la vinculación entre lo que se llama violencia estructural con la violencia entre pares dentro de las aulas, Bourdieu (2008) identifica la institución educativa como un aparato que reproduce las formas de vinculación mediadas por una jerarquía de poderes, lo que supondría la base de las relaciones de dominación y violencia. Además, el autor propone el reconocimiento de las leyes de reproducción como base para su minimización.

La "institución reproductora" de Bourdieu (2008), la "institución disciplinaria"

$6 \quad$ Paredes, Álvarez, Lega y Vernon (2008) se refieren a bullying como un sinónimo de acoso escolar. Las mismas autoras reconocen que el término en inglés ha sustituido el uso de sus sinónimos en español en la literatura sobre el tema, con acepciones diferentes. Una distinta conceptualización se asocia con el uso del vocablo inglés. 
o panóptico de Foucault (2005), el "aparato ideológico del Estado" de Althusser (1989), la "institución total" de Goffman (2006): no se puede desvincular el carácter normalizador de la escuela en relación a sus instituciones homólogas: la prisión, el hospital psiquiátrico, el orfanato, el asilo para ancianos, el ejército.

Se encuentra entonces ante una escuela que se proyecta como un gran aparato podador de subjetividades $y$ verdugo de diferencias $y$ diversidades, creador de antemano de esta noción de diversidad, del Otro distinto, alienado, dicho sea de paso.

D’Antoni y Gómez (2014), hacen una problematización del concepto bullying —en inglés y como fenómeno de importación-y a su vez hacen una vinculación entre la violencia estructural, así como, la influencia del autoritarismo en la proliferación y afirmación de las relaciones de poder.

Existe también una tendencia a abordar el fenómeno del bullying por medio de la creación o caracterización compulsiva de los "perfiles del bully" (o agresor), este enfoque también está presente en el abordaje de temáticas como el abandono escolar, o "expulsión estructural o sistemática” (D’Antoni, Gómez, Gómez y Soto, 2013). González (2006) explica que una gran parte del material sobre abandono o absentismo escolar versa sobre el individuo que abandona, creando perfiles, características asociadas e identificando factores de riesgo, al respecto, la autora critica el hecho de que este enfoque deja por fuera aspectos sociales, políticos y organizativos.

\section{DISCUSIÓN}

En este taller se reflejó la visión "tradicional" de lo que la investigación ha definido como bullying. La violencia verbal, el choteo, las humillaciones y las bromas con doble sentido originan dolor y pueden ser una de las causas o concausas del abandono escolar.

También emerge del taller un afecto o una visión protectora del colegio nocturno, percibido como un lugar seguro, una referencia cuando otras modalidades han sido expulsoras. Los y las participantes describen la institución como menos problemática que otras: el estudiantado es adulto y está en capacidad de defenderse.

Entre las causas que provocan la violencia se encuentran la drogadicción, el machismo y el alcoholismo, asimismo, existe conciencia de que entre estas hay una relación. Sin embargo, permanece incipiente la posibilidad de mapearlas y la constitución de modelos explicativos articulados en la representación que se tiene de la violencia. Por otro lado, es evidente la ubicación de la violencia en la historia personal y su vinculación con la exclusión educativa.

El colegio nocturno aparece como una institución menos violenta; no obstante, no es inmune de los problemas sociales y contextuales.

Se considera pertinente traer a colación la discusión del fenómeno de abandono de las aulas vinculado con el bullying, en vista de que una visión simplista o plana del fenómeno de la violencia se podría interpretar como un acto de evasión por parte del sistema educativo, como la incapacidad de conseguir una respuesta institucional que enfrente estas problemáticas: es más conveniente encontrar una explicación que ponga la responsabilidad sobre la voluntad de los y las estudiantes que sobre las insuficiencias de la institución.

La discusión que se pretende abordar a partir de las reflexiones de D'Antoni y Gómez (2013), surge de la interrogante sobre qué significa para las altas cúpulas administradoras de la educación costarricense, el hecho de que se emplee un término prestado $y$ vinculado con una cultura ajena a la nuestra. Asimismo, es oportuno problematizar por qué existe tan poca investigación respecto al análisis de la violencia en las aulas como un síntoma de la violencia estructural que se ejerce en el sistema educativo como aparato excluyente en sí.

\section{BIBLIOGRAFÍA}

\section{LIBROS}

Althusser, Louis (1970). La ideología y los aparatos ideológicos del Estado.18a. edición. México: Siglo xxI, 1989.

Apple, Michael (1928). Ideología y currículo. Madrid: Akal Ediciones, 2008. 
Baquero, Ricardo. "De las dificultades de predecir: educabilidad y fracaso escolar como categorías riesgosas". Construyendo posibilidad. Apropiación y sentido de la experiencia escolar. R. Baquero, A. Pérez y A. Toscano. (compiladores). Rosario, Argentina: Homo Sapiens, 2009.

Bordieu, Pierre (1997). Capital cultural, escuela y espacio simbólico. 2da. edición. Buenos Aires, Argentina: Siglo xxI, 2008.

D' Antoni, Maurizia; Gómez Ordoñez, Luís; Gómez Torres, Juan y Soto Arguedas, José Fabio. La escuela en cuestionamiento: diálogos problematizadores sobre la resistencia escolar en procesos pedagógicos emergentes. San José, Costa Rica: Editorial Arlekín, 2013.

Foucault, Michel (1975). Sorvegliare e punire. Nascita della prigione. Turín, Italia: Einaudi, 2005.

Goffman, Erving (1963). Estigma La identidad deteriorada. $10 \mathrm{ma}$. Buenos Aires, Argentina: Amorrortu Editores, 2006.

\section{PUBLICACIONES PERIÓDICAS}

Baquero, Ricardo. "La educabilidad bajo sospecha". Cuaderno de Pedagogía 9. Rosario, Argentina. Octubre 2001: 71-85. En: http://www.porlainclusionmercosur.educ. ar/ documentos/educabilidadCuadernosBaquero.pdf [consultado el 20 de enero de 2014].

Calvo Salvador, Adelina y Susinos Rada, Teresa. “'Yo no valgo para estudiar...': un análisis crítico de la narración de las experiencias de exclusión social". Contextos Educativos 8-9. Cantabria, España. Universidad de Cantabria, 2005-2006: 87-106. [Revista digital] En: <http://publicaciones.unirioja.es/ ojs-2.4.2/index.php/contextos/article/ view/559> [consultado el 18 de enero de 2014].

D' Antoni, Maurizia y Soto Arguedas, José Fabio. "Experiencias de investigación en la secundaria. Una visión desde adentro". Temas de Nuestra América. Revista de Estudios Latinoamericanos 49 (1).
Heredia, Costa Rica: Instituto de Estudios Latinoamericanos de la Universidad Nacional de Costa Rica, 2010.

García Rivera, Enrique. "Reflexionando en torno a la investigación educativa: una mirada crítica desde la auto etnografía de un docente". Qualitative Research in Education 1 (1). España. Universidad de Granada, 2012: 58-79. [Revista digital]. En: <http://dialnet.unirioja.es/servlet/ articulo?codigo $=4040006>$ [consultado el 27 de enero de 2014].

Gómez, Susana; Leigh, Camila y Nova, Yolanda. "Reflexiones sobre como el curriculum escolar genera desigualdad y violencia: una propuesta para el cambio en la escuela”. Estudios Hemisféricos y Polares 3 (3). Chile. Universidad de Playa Ancha (UPLA), 2012: 174-193.

González González, María Teresa. "Absentismo y abandono escolar: una situación singular de la exclusión educativa". Revista electrónica Iberoamericana sobre Calidad, Eficacia y Cambio en Educación 4. España. Red Iberoamericana de Investigación sobre Cambio y Eficacia Escolar (RINACE), 2006: 1-15. [Revista digital] En: <http://www. rinace.net/arts/vol4num1/art1.htm > [consultado el 10 de junio de 2014].

Guedes, Alessandra; García-Moreno, Claudia y Bott, Sarah. "Violencia contra las mujeres en Latinoamérica y el Caribe”. Foreign Affairs Latinoamérica 1 (14). México. Instituto Tecnológico Autónomo de México (ITAM). 2014: 41-8.

Paredes, María Teresa; Álvarez, Martha Cecilia; Lega, Lonor I. y Vernon, Ann. "Estudio exploratorio sobre el fenómeno del "bullying" en la ciudad de Cali, Colombia". Revista Latinoamericana de Ciencias Sociales, Niñez y Juventud 6. Colombia, 2008: 295-317. [Revista digital]. En: <http://revistaumanizales. cinde.org.co/index.php/RevistaLatinoamericana/article/view/276/142> [consultado el 10 de junio de 2014].

Rivera C, Ernesto. "Encuesta del CIEP para UnIVERSIDAD: Pesimismo envuelve 
rumbo del país y gestión del Gobierno". Semanario Universidad. San José, Costa Rica: Universidad de Costa Rica. 8 de mayo de 2013. En: <http:// semanariouniversidad.ucr.cr/pais/ encuesta-del-ciep-para-universidadpesimismo-envuelve-rumbo-del-pas-ygestin-del-gobierno/> [consultado el 7 de junio de 2015].

Salinas Comboni, Sonia. "Interculturalidad, educación y política en América Latina”. Política y cultura 17. México. Universidad Xochimilco, 2002: 261-287. [Revista digital] En: <http://dialnet.unirioja. es/servlet/articulo?codigo $=4151809>$ [consultado el 27 de enero de 2014].

\section{TEXTOS ELECTRÓNICOS}

Gutiérrez, Susana. Choteo, característica sociocultural del ser costarricense. Huellas culturales. Interpretación textual de la construcción de identidades culturales. 2013. En: <http://huellasculturales11. wordpres s.com/trabajos-deestudiantes-2013/susana-gutierrez/> [consultado el 10 de junio de 2014].

Programa Estado de la Nación. Decimosexto Informe Estado de la Nación en Desarrollo Humano Sostenible. San José, Costa Rica: Programa Estado de la Nación. 2010. En: <http://www. estadonacion.or.cr/> [consultado el 27 de enero de 2014].
Programa Estado de la Nación. Tercer informe Estado de la Educación. San José, costa Rica: Programa Estado de la Nación. 2011. En: <http://www.estadonacion. or.cr/estado-educacion/educacion-porcapitulo/educacion-informe-ultimo> [consultado el 27 de enero de 2014].

Programa Estado de la Nación. Cuarto Informe Estado de la Educación. San José, Costa Rica: Programa Estado de la Nación. 2013. En:<http://www. estadonacion.or.cr/estado-educacion/ educacion-informe-ultimo $>$ [consultado el 27 de enero de 2014].

OTROS

D’Antoni, Maurizia y Gómez Ordoñez, Luís. "Violencia, bullying y autoritarismo en la escuela secundaria". [Ponencia II Encuentro de Psicología Comunitaria]. Heredia, Costa Rica. 22-24 de mayo, 2013.

Leiva Picado, Esteban. IPEC-CINDEA es fuente de conocimiento y superación. $\mathrm{Mi}$ Cartago. 12 de noviembre de 2009. En: $<$ http://www.micartago.com/index. php? news $=1404>$ [consultado el 26 de enero de 2014].

Fecha de ingreso: 03/03/2014 Fecha de aprobación: 14/08/2014 